\title{
Closed-Cage Clusters in the Gaseous and Condensed Phases Derived from Sonochemically Synthesized $\mathrm{MoS}_{2}$ Nanoflakes
}

\author{
D. M. David Jeba Singh, ${ }^{a}$ T. Pradeep, ${ }^{a}$ Joydeep Bhattacharjee, ${ }^{b}$ \\ and Umesh V. Waghmare ${ }^{\mathrm{b}, *}$ \\ a Department of Chemistry and Sophisticated Analytical Instrument Facility, Indian Institute of Technology \\ Madras, Chennai, India \\ b Theoretical Sciences Unit, Jawaharlal Nehru Centre for Advanced Scientific Research, Jakkur PO, Bangalore, \\ India
}

The $\mathrm{Mo}_{13}$ clusters we previously reported were derived from $\mathrm{MoS}_{2}$ flakes prepared from bulk $\mathrm{MoS}_{2}$, although the nature of the precursor species was not fully understood. The existence of the clusters in the condensed phase was a question. Here we report the preparation of $\mathrm{MoS}_{2}$ nanoflakes from elemental precursors using the sonochemical method and study the gas-phase clusters derived from them using mass spectrometry. Ultraviolet-visible (UV-vis) spectrum of the precursor is comparable to nano $\mathrm{MoS}_{2}$ derived from bulk $\mathrm{MoS}_{2}$. High-resolution transmission electron microscopy (HRTEM) revealed the formation of nanoflakes of $\mathrm{MoS}_{2}$ with 10to 30-nm length and 3- to 5-nm thickness. Laser desorption ionization mass spectrometry (LDI-MS) confirmed the formation of $\mathrm{Mo}_{13}$ clusters from this nanomaterial. Matrix-assisted laser desorption ionization mass spectrometry (MALDI-MS) points to the existence of $\mathrm{Mo}_{13}$ clusters in the condensed phase. The clusters appear to be stable because they do not fragment in the mass spectrometer even at the highest laser intensity. Computational analysis based on generalized Wannier orbitals is used to understand bonding and stability of the clusters. These clusters are highly stable with a rich variety in terms of centricity and multiplicity of Mo-Mo, S-Mo, and S-S bonds. (J Am Soc Mass Spectrom 2007, 18, 2191-2197) (C) 2007 American Society for Mass Spectrometry

$\mathrm{D}$ iscovery of fullerenes, closed-cage clusters of carbon, motivated researchers to explore the possibility of finding similar clusters from elements other than carbon. The discovery of metallocarbohedrenes [1,2] added greater impetus to this effort. The formation of inorganic fullerenes [3] from layered compounds like metal dichalcogenides $\mathrm{MX}_{2}(\mathrm{M}=\mathrm{Mo}$, $\mathrm{W} ; \mathrm{X}=\mathrm{S}, \mathrm{Se})$ and their application in the field of tribology [4] generated further interest. There are several synthetic approaches such as gas-phase deposition $[5,6]$, laser ablation $[7,8]$, electron irradiation [9], and high-temperature chemical routes $[10,11]$ for the synthesis of clusters. Nano forms of these materials have their own advantages over the corresponding bulk materials. Electrochemical deposition [12] and sonochemical routes $[13,14]$ are some other methods adapted for the synthesis of nanostructured materials. Electrochemical methods result in the formation of

Address reprint requests to Prof. T. Pradeep, Indian Institute of Technology Madras, Department of Chemistry and Sophisticated Analytical Instrument Facility, Chennai, Tamil Nadu 600 036, India. E-mail: pradeep@iitm.ac.in,or Prof. Umesh V. Waghmare, Theoretical Sciences Unit, Jawaharlal Nehru Centre for Advanced Scientific Research, Jakkur PO, Bangalore 560 064, India. E-mail: waghmare@jncasr.ac.in nanomaterials as a film, whereas sonochemical methods produce free-standing nanoflakes of metal chalcogenides with controlled size. A study by Mdleleni et al. [15] reported a route for the preparation nanostructured $\mathrm{MoS}_{2}$ by a simple sonochemical method. In that report, $\mathrm{Mo}(\mathrm{CO})_{6}$ and sulfur as elemental precursors were used in isodurene as the solvent. Sonochemical syntheses of molybdenum oxide and molybdenum carbide were reported by Dhas and Gedanken [16]. Sostaric et al. [17] made colloidal CdS by this route. Fullerene-like inorganic structures were made by the sonochemical treatment of aqueous $\mathrm{TlCl}_{3}$ by Avivi et al. [18]. $\mathrm{MoS}_{2}$ clusters in the gas phase have been the subject of investigation by a few research groups [19-23]. James et al. [19] reported $\mathrm{Mo}-\mathrm{C}, \mathrm{Mo}-\mathrm{S}$, and $\mathrm{Mo}-\mathrm{N}$ cluster formation in the gas phase. Mo and $\mathrm{W}$ disulfide nanoplatelets in the gas phase have been studied by Bertram et al. [20]. Gemming et al. [21] explored the small metal sulfide $\mathrm{M}_{\mathrm{x}} \mathrm{S}_{\mathrm{y}}(\mathrm{M}=\mathrm{Mo}, \mathrm{W} ; \mathrm{x}=1,2,4 ; \mathrm{y}=1-12)$ clusters using theoretical calculations and mass spectrometry. There have been noteworthy investigations on catalysis of nano- $\mathrm{MoS}_{2}$ using scanning tunneling microscopy (STM) and associated techniques. Recently Lauritsen et al. [24] studied the variation in electronic structure with respect 
to the size and morphology of $\mathrm{MoS}_{2}$ nanoclusters. Formation of nanooctahedra of $\mathrm{MoS}_{2}$ and $\mathrm{MoSe}_{2}$ using pulsed laser vaporization was also investigated [25].

Recently in a letter [26], we reported the formation of closed-cage clusters in the gas phase, derived from $\mathrm{MoS}_{2}$. The parent material was derived from bulk $\mathrm{MoS}_{2}$ by ball milling. The separated nanoflakes of $\mathrm{MoS}_{2}$ were analyzed by laser desorption ionization mass spectrometry (LDI-MS) and characterized by spectroscopic techniques. Structures of these clusters were studied using computations and were found to be similar to the single-layered inorganic fullerenes. The nature of the precursor and the existence of magic clusters in the condensed phase are two important questions we sought to answer. In this paper, we prepared these clusters from sonochemically synthesized nano-MoS ${ }_{2}$, starting from elemental precursors. The nature of the parent material was established by high-resolution transmission electron microscopy (HRTEM). The presence of these clusters in the condensed phase was investigated by matrix-assisted laser desorption ionization mass spectrometry (MALDI-MS). This paper is a continuation of our investigations into the structure and stability of metal chalcogenides and metal oxides in the gas phase [27, 28].

\section{Experimental}

Sonochemical treatment of a mixture of $\mathrm{Mo}(\mathrm{CO})_{6}$ and sulfur (1:2 Mo:S ratio) in o-xylene for 3-4 $\mathrm{h}$ yielded nano $\mathrm{MoS}_{2}$. The concentration of $\mathrm{Mo}(\mathrm{CO})_{6}$ was $0.1 \mathrm{M}$ and typical volume used for synthesis was $10 \mathrm{~mL}$. During the synthesis, the solution, originally colorless, became blue. The nanoflakes were centrifuged out and were washed several times with o-xylene before analysis. A black redispersible solid material was obtained at the end and was stored in this state. The formation of nano-MoS ${ }_{2}$ has been confirmed from the UV-visible spectrum which was comparable to our earlier results [26]. Figure 1 shows the ultraviolet-visible (UV-vis) diffused reflectance spectrum of bulk $\mathrm{MoS}_{2}$ [trace a] and synthesized nano-MoS $\mathrm{M}_{2}$ powder [trace b]. The peaks at 598 and $648 \mathrm{~nm}(2.08$ and $1.92 \mathrm{eV})$ correspond to the $\mathrm{B}_{1}$ and $A_{1}$ exciton peaks, respectively. These bands have been attributed to the direct transfer at the $\mathrm{K}$ point of the Brillouin zone [29]. The spacing between these two exciton peaks is $0.16 \mathrm{eV}$, which corresponds to $\mathrm{MoS}_{2}$, and confirms the formation of $\mathrm{MoS}_{2}$. This exciton corresponds to the smallest direct or characteristic gap between valence and conductance bands. The exciton separation is due to the spin-orbit splitting of the valence band at the $\mathrm{K}$ point. Above a particle size of $8-10 \mathrm{~nm}$, the peaks are at bulk values [30]. This agrees with the transmission electron microscopy (TEM) measurements (see following text).

For certain experiments, nano- $\mathrm{MoS}_{2}$ derived from bulk $\mathrm{MoS}_{2}$ was irradiated with UV light from a 400-W $\mathrm{Hg}$ arc lamp (Oriel Instruments, Stratford, CT, USA), until the color changed from blue to green. The sample

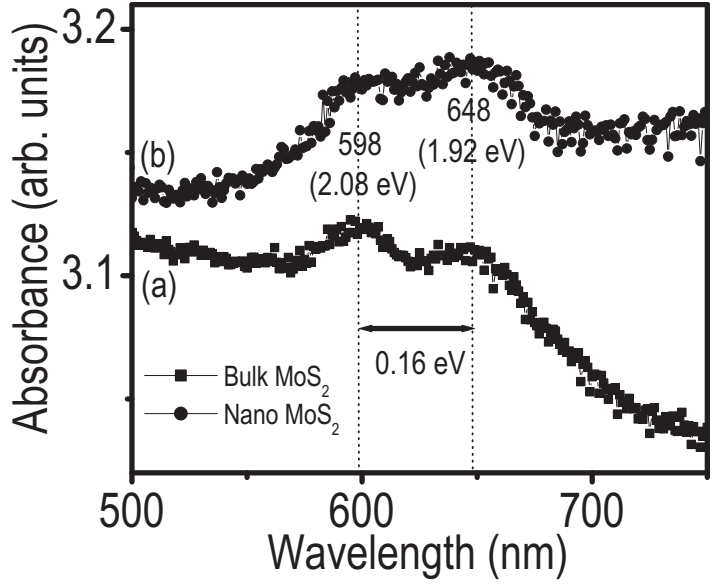

Figure 1. UV-visible diffused reflectance spectra of bulk $\mathrm{MoS}_{2}$ (a) and synthesized $\mathrm{MoS}_{2}$ (b).

was irradiated in a quartz tube for $2 \mathrm{~h}$. The UVirradiated sample was further studied by LDI.

The prepared material was investigated by dispersing it in o-xylene and spotting on the target plate of a Voyager DE PRO Biospectrometry Workstation (Applied Biosystems, Foster City, CA, USA) MALDI-TOF (time of flight) MS instrument. A film was formed after drying the dispersion. For MALDI-MS analysis the samples were dispersed in appropriate matrices in 1:10 ratio (by volume). A pulsed nitrogen laser of $337 \mathrm{~nm}$ was used and the TOF was operated in the delayed extraction mode. For fragmentation by post source decay (PSD), we used a timed ion selector. The theoretical mass spectra were simulated using Data Explorer version 4.0.0.0 software provided by Applied Biosystems, which uses the isotope database of IUPAC [31]. The isotope pattern was clearly discernible in all the cluster peaks and a comparison of the expected and observed patterns was used to assign the peaks.

Most of the measurements were done in the linear TOF mode. All measurements corresponded to the negative-ion mode. For fragmentation as well as clustering studies, we used the timed ion selector by which we can select a particular $\mathrm{m} / \mathrm{z}$ and study its metastable decay. The mass selection in this mode has an uncertainty of $\pm 4 \mathrm{Da}$.

\section{Computational Details}

To confirm the stability of the observed clusters and to understand their chemical origin, we carried out detailed studies of their energetics and bonding based on nonempirical calculations [32] of the quantum mechanical ground state of electrons using the density functional theory (DFT) with a generalized gradient approximation to the many electrons exchange correlation energy given by the Perdew-Burke-Ernzerhof (PBE) functional [33]. We used a standard plane-wave code PWSCF 2.0.1 with ultrasoft pseudopotentials [34] to represent the interaction between the ions and elec- 
trons. An energy cutoff of 25 Rydberg units (Ry) was used on the plane-wave basis used in the representation of wave functions; the cutoff was $150 \mathrm{Ry}$ for the representation of density. Structures of clusters were determined through total energy minimization using the Broyden-Fletcher-Goldfarb-Shanno (BFGS) algorithm and Hellmann-Feynman forces on atoms. In this procedure, we used different polyhedral structures as initial guesses for smaller clusters and various cage structures of sulfur atoms with Mo atoms located on an inner shell at positions with four- to sixfold sulfur coordination as initial guesses for larger clusters. A large supercell was used with periodic boundary conditions with a vacuum of at least $10 \AA$ separating periodic images and a single k-point $(0,0,0)$ as Bloch vector.

Ground-state electron charge density and corresponding Kohn-Sham orbitals (which are energy eigenfunctions) emerging from the density functional theory calculations are not directly useful in obtaining a precise picture of bonding. Here, we use a recently developed [35] generalized Wannier orbital approach in the analysis of bonding in these clusters. Although Wannier orbitals have been extensively used in studies of extended periodic systems, they have not been commonly used in studies of confined systems such as clusters. These orbitals are localized in real space and provide a description of electronic states that readily relates to the nature of bonding. For crystals, they are obtained as a Fourier transform of extended Bloch functions that are smooth as a function of Bloch vector $\mathrm{k}$. In the case of clusters, as described in Baroni et al. [32], generalized Wannier orbitals can be obtained by simultaneously diagonalizing $\mathrm{x}, \mathrm{y}$, and $\mathrm{z}$ operators in the occupied subspace (this can be done only approximately because these operators do not commute with each other when projected into the occupied subspace). A standard output of a Wannier function analysis consists of (1) positions of the bond centers and (2) localized bonding orbitals (centered at these positions), whose shape readily reveals the contribution of different atomic orbitals to bonding. Count of Wannier centers between two or more nearest-neighbor atoms give the bond order. A multicentered bond is naturally identified if the orbital is centered inside a polyhedron of a few atoms. These orbitals, as implemented in a plane-wave DFT code ABINIT [36], were used in the present analysis.

\section{Results and Discussion}

The synthesized nano- $\mathrm{MoS}_{2}$ material was characterized using high-resolution transmission electron microscopy (HRTEM). The HRTEM analysis revealed (Figure 2) the formation of nanoflakes of 10-20 nm width. Other geometries, in addition to the elongated flakes, were also found. The flakes have bulk $\mathrm{MoS}_{2}$ structure as revealed by the electron-diffraction pattern from the whole area. The inset of Figure 2 shows a hexagonal

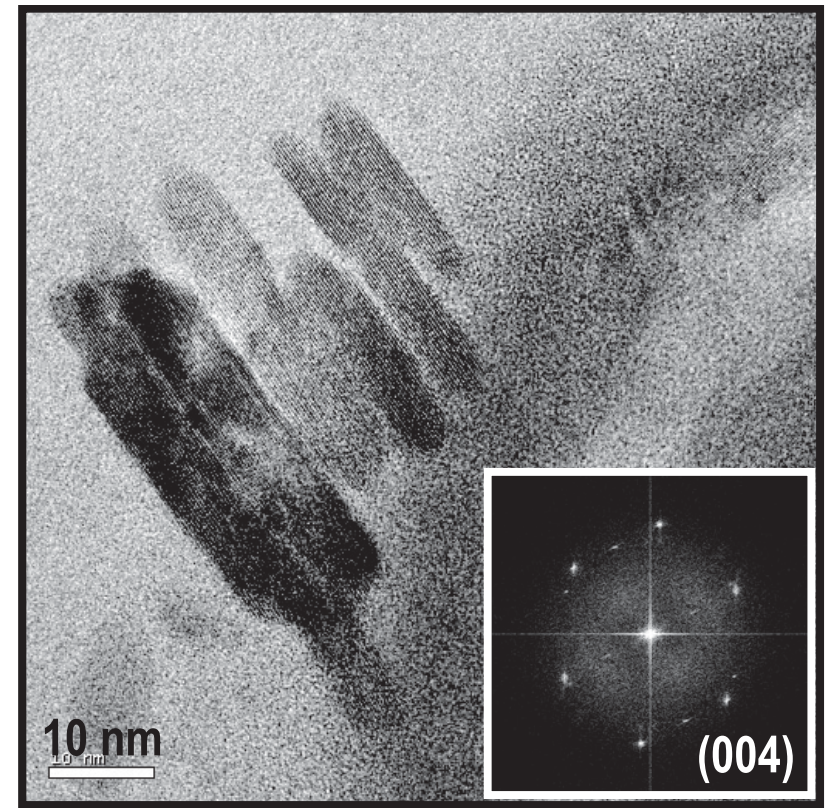

Figure 2. HRTEM image of the synthesized nano $\mathrm{MoS}_{2}$ flakes. Stacked nanoflakes are seen. Inset: Fourier transform of the image showing a hexagonal pattern.

pattern corresponding to bulk $\mathrm{MoS}_{2}$. A spacing of $3.1 \AA$ is seen in the platelets corresponding to the (004) plane of $\mathrm{MoS}_{2}$ (PCPDF No. 87-2416). Surface investigations of $\mathrm{MoS}_{2}$ nanorods have also reported an inter-planar spacing of about $3.1 \AA$ [37]. Upon investigation of an edge of one such nanoflake, we observe a cubic pattern (Supplementary material Figure S1, which can be found in the electronic version of this article). The spacing seen at the edge, although similar to the (004) plane of $\mathrm{MoS}_{2}$, appears to be due to the (111) plane of cubic $\mathrm{Mo}_{3} \mathrm{O}$ (PCPDF No. 72-0527). The molybdenum-rich oxide material may form by the aerial oxidation of the metallic molybdenum intermediate formed during the course of the reaction. It has been suggested that $\mathrm{Mo}(\mathrm{CO})_{6}$ decomposes first to form Mo nanoflakes, which further react with sulfur to form $\mathrm{MoS}_{2}$ [10]. There are no other cubic phases seen in oxides or sulfides of Mo.

A negative-mode LDI mass spectrum of the synthesized nano- $\mathrm{MoS}_{2}$ nanoflakes sample is shown in Figure 3. Isotope patterns of the peaks were compared with the expected pattern for a given molecular formula and this was used to assign the peaks. Even in very pure samples of $\mathrm{MoS}_{2}$, oxygen added $\mathrm{M}_{\mathrm{n}} \mathrm{S}_{\mathrm{m}} \mathrm{O}^{-}$and $\mathrm{M}_{\mathrm{n}} \mathrm{O}_{\mathrm{y}}$ clusters were observed due to possible oxidation of the edges of the nanoplates. These peaks may also be attributed to the chemical reactivity of the clusters with residual oxygen. This latter possibility is unlikely in a vacuum of $10^{-8}$ torr present in the mass spectrometer. Therefore, we attribute their presence to oxidized nanosheets. In the lower mass range, the clusters formed are $\mathrm{MoS}_{2}{ }^{-}, \mathrm{MoS}_{3}{ }^{-}, \mathrm{MoS}_{4}{ }^{-}, \mathrm{Mo}_{2} \mathrm{~S}_{3}{ }^{-}, \mathrm{Mo}_{2} \mathrm{~S}_{5}{ }^{-}$, $\mathrm{Mo}_{2} \mathrm{~S}_{6}{ }^{-}, \mathrm{Mo}_{2} \mathrm{~S}_{8}{ }^{-}$, and so forth. Isotope patterns of these peaks matched exactly. The spectrum exhibits a trend 


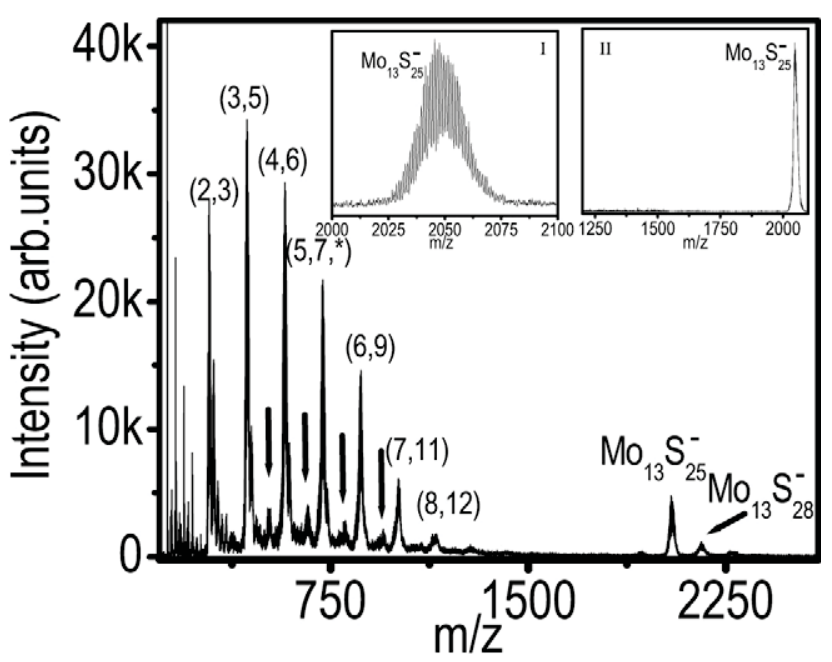

Figure 3. LDI mass spectrum of synthesized $\mathrm{MoS}_{2}$ nanoflakes in the negative mode. Peaks $(\mathrm{m}, \mathrm{n})$ correspond to $\mathrm{Mo}_{\mathrm{m}} \mathrm{S}_{\mathrm{n}}$. Peak at $\mathrm{m} / \mathrm{z}$ 720 (labeled *) is attributed to oxygen addition on $\mathrm{Mo}_{5} \mathrm{~S}_{7}$. Arrows indicate other oxygen-containing ions. Inset I: Expanded view of $\mathrm{Mo}_{13} \mathrm{~S}_{25}{ }^{-}$showing its isotope distribution. Inset II: PSD mode LDI mass spectrum of $\mathrm{Mo}_{13} \mathrm{~S}_{25}{ }^{-}$showing no fragmentation. The PSD data were collected in four separate regions and stitched together later.

with the intensity increasing from $\mathrm{MoS}_{2}{ }^{-}$, reaches a maximum around $\mathrm{Mo}_{3} \mathrm{~S}_{5}{ }^{-}$, and decreases thereafter. Some of the ions are found to add oxygen preferentially as in the case of $\mathrm{Mo}_{5} \mathrm{~S}_{7}$, although the details of such addition products were not investigated in this work.

Beyond this region, the spectral intensity decreases uniformly until $\mathrm{m} / \mathrm{z} 2000$ and a magic peak appears with a maximum at $m / z 2049$, followed by another peak at $\mathrm{m} / \mathrm{z}$ 2144. These peaks are at positions identical to our earlier report [26]. We note that no peaks between $\mathrm{m} / \mathrm{z}$ 2049 and 2144 are detected, unlike in the case of lower mass number region (up to $\mathrm{m} / \mathrm{z} 1000$ ). Therefore, we suggest that the ions at $\mathrm{m} / \mathrm{z} 2049$ and 2144 do not contain oxygen. An expanded mass spectrum of the $\mathrm{Mo}_{13} \mathrm{~S}_{25}{ }^{-}$is shown in inset I. The magic peak at $\mathrm{m} / \mathrm{z} 2049$ corresponding to $\mathrm{Mo}_{13} \mathrm{~S}_{25}{ }^{-}$was further analyzed by PSD to see its stability. Even at the highest laser power available, no observable fragments were obtained as shown in inset II. Therefore, the structure is likely to be a stable one.

Until now we discussed the formation of clusters in the gas phase by the LDI method. The MALDI-MS analyses of this material with various matrices show some interesting results. We tried matrices such as cyano-4-hydroxycinnamic acid (CHCA), sinapinic acid (SA), and trans-3-indoleacrylic acid (IAA). In all these matrices, the results are similar with small intensity differences in the peaks. The MALDI-MS analyses of these materials (Figure 4 ) reveal the possibility of existence of a molecular compound with the $\mathrm{Mo}_{13}$ core. MALDI spectra show that the $\mathrm{Mo}_{13}$-containing peaks are present, whereas intensities of all the other small clusters, which were shown up in LDI analysis, fade or even vanish. The preferential enhancement in the $\mathrm{Mo}_{13}$

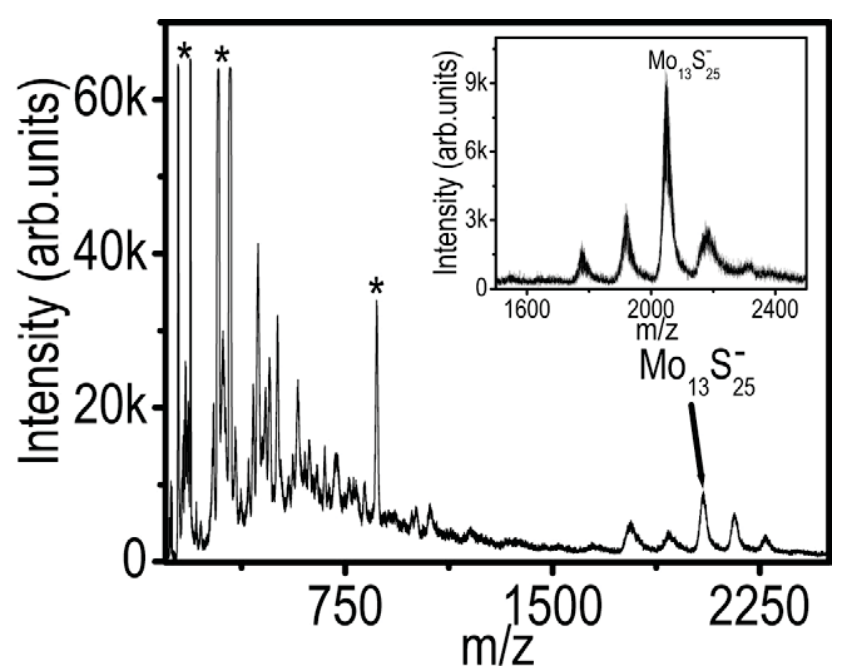

Figure 4. MALDI mass spectrum of the synthesized $\mathrm{MoS}_{2}$ nanoflakes with CHCA matrix in the negative ion mode showing the magic clusters. Lower mass range showing matrix-related peaks along with smaller clusters. The peaks marked with asterisks $\left(^{*}\right)$ are attributed to the matrix. Inset shows the MALDI mass spectrum of synthesized $\mathrm{MoS}_{2}$ nanoflakes with IAA matrix in the region of interest.

peaks suggested that a molecular entity could be existing in the sample. It may be noted that the $\mathrm{Mo}_{13}$ peak is stronger than those of the neighboring clusters. These ions were also analyzed by PSD and no features due to fragments were observed.

Polyoxomolybdates are well known to form giant clusters in the solid state. To check the possibility of these clusters in the gas phase and also to compare our

(a)
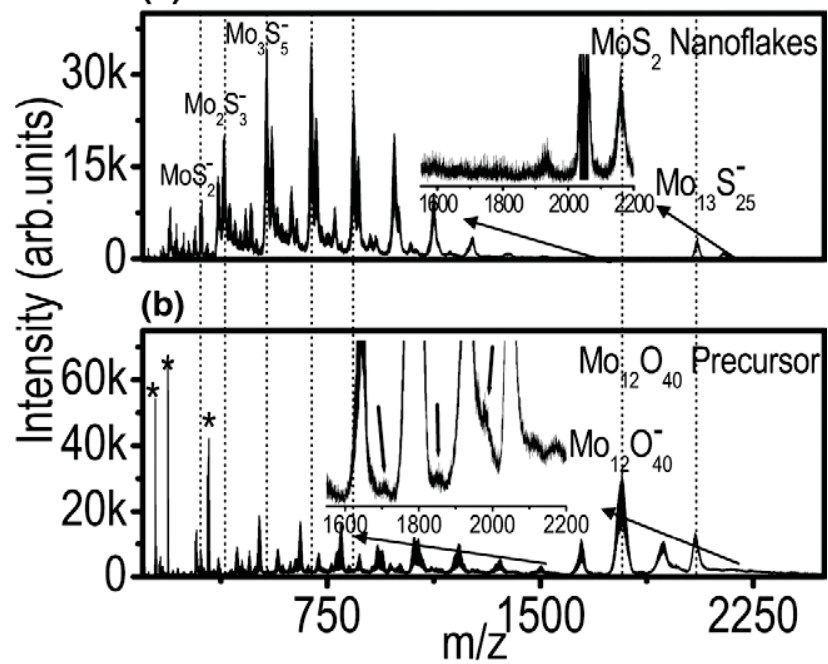

Figure 5. Comparison between the $\mathrm{Mo}_{13} \mathrm{~S}_{25}$ clusters obtained in LDI (derived from $\mathrm{MoS}_{2}$ nanoflakes synthesized from elements) (a) with the clusters obtained in MALDI of the $\mathrm{Mo}_{12} \mathrm{O}_{40}$ precursor in CHCA matrix (b). In (b) the enlarged high mass region shows peaks in-between the magic clusters (marked with arrows). The $\mathrm{Mo}_{13}$ region of (a) is also expanded in the same mass window. The matrix peaks in $(\mathbf{b})$ are labeled with asterisks $\left(^{*}\right)$. The dotted lines are intended to show the distinct nature of the $\mathrm{MoS}_{2}$ clusters. 
clusters with those possible with oxygen (as mass of sulfur can be due to two oxygens), we tried MALDI-MS analysis of $\mathrm{H}_{3} \mathrm{PMo}_{12} \mathrm{O}_{40}$ with various matrices and found a number of clusters (Figure 5). The lower mass range peaks have successive addition of $\mathrm{MoO}_{3}$ and $\mathrm{MoO}_{4}$ moieties, which build up the larger clusters. This kind of cluster formation in the gas phase by molybdenum oxide has been reported earlier [28]. We note that these clusters are significantly different with a base peak due to $\mathrm{Mo}_{12} \mathrm{O}_{40}$ at $\mathrm{m} / \mathrm{z} 1790$ (due to the parent ion, pre-existing in the solid state). There is an ion at $\mathrm{m} / \mathrm{z}$ 2049 , but this has a mass difference of $\mathrm{MoO}_{3}$ with the adjacent clusters. The spectral pattern is also distinctly different in this case. In Figure 5 we compare the LDI-MS spectrum of the synthesized $\mathrm{MoS}_{2}$ nanoflakes along with the MALDI spectrum of the $\mathrm{Mo}_{12}$ precursor, where we can clearly see the distinct differences. It is important to note that there are peaks in the in-between mass region in the oxide-derived clusters. For example, between every magic cluster peak, there are ions (see the enlarged spectrum in Figure 5B; the ions in-between are marked). It is important to note that there were no ions in LDI in this case and spectra could be collected only in MALDI. Only pre-existing ions could be studied. Similar results have been achieved using other matrices such as IAA with small intensity differences in the peaks.

In continuation of our earlier work [26] on the $\mathrm{Mo}_{13}$ cluster, we have done additional calculations exploring a few more possible structures. In the case of large clusters, $\mathrm{Mo}_{13} \mathrm{~S}_{25}$ and $\mathrm{Mo}_{14} \mathrm{~S}_{25}$ are more stable than other clusters having similar mass, for example, $\mathrm{Mo}_{13} \mathrm{~S}_{28}$. The optimized structure of $\mathrm{Mo}_{14} \mathrm{~S}_{25}$ is very similar to that of $\mathrm{Mo}_{13} \mathrm{~S}_{25}$ with an additional Mo atom inside the cage, which otherwise encloses an almost spherical void space [26] with a diameter of about $4.5 \AA$ A. The cage is formed by a core of Mo atoms each with 4-6 neighboring $S$ atoms located in an outer shell. Each $S$ atom in the outer shell has 1-3 neighboring Mo atoms. Both $\mathrm{Mo}_{13} \mathrm{~S}_{25}$ and $\mathrm{Mo}_{14} \mathrm{~S}_{25}$ structures have threefold symmetry. We find that $\mathrm{Mo}_{14} \mathrm{~S}_{25}$ is more stable than $\mathrm{Mo}_{13} \mathrm{~S}_{28}$ (of roughly the same mass) by about $0.4 \mathrm{eV}$ per atom.

The $\mathrm{Mo}_{14}$ cluster was found with increased intensity in the LDI-MS analysis of UV-irradiated $\mathrm{MoS}_{2}$ nanoflakes. The UV irradiation was done to see whether the smaller cluster present in the solid state (as revealed by MALDI-MS) undergoes any further change, given that molybdenum clusters are known to transform to distinct chemical forms upon photoirradiation [38]. The protocol followed for photoirradiation was as previously described [26]. The LDI spectrum (Figure 6) of the UV-irradiated product gave the magic cluster as the base peak. We also note that the spectrum is distinctly different, with the ion at $m / z 2144$ becoming the base peak. We had assigned these peaks to $\mathrm{Mo}_{13} \mathrm{~S}_{25}{ }^{-}$and $\mathrm{Mo}_{13} \mathrm{~S}_{28}{ }^{-}$in our earlier report. The second ion is more likely to be $\mathrm{Mo}_{14} \mathrm{~S}_{25}{ }^{-}$rather than $\mathrm{Mo}_{13} \mathrm{~S}_{28}{ }^{-}$from the fragmentation studies. The PSD analysis, presented in the inset of Figure 6, shows no fragmentation, suggest-

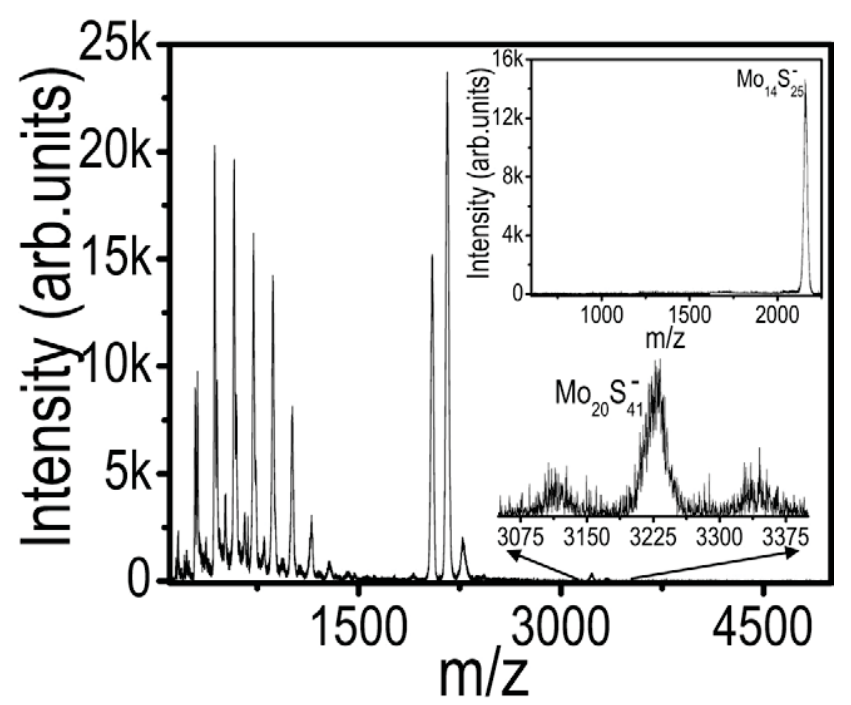

Figure 6. LDI mass spectrum of derived $\mathrm{MoS}_{2}$ nanoflakes after UV irradiation in the negative mode showing the magic number cage clusters. A weak peak attributed to a $\mathbf{M o}_{20}$ cluster is also shown. Enlarged peak of $\mathrm{Mo}_{20} \mathrm{~S}_{41}$ is also shown, above the spectrum. Inset: PSD mode LDI mass spectrum of $\mathrm{Mo}_{14} \mathrm{~S}_{25}$ showing no fragmentation. The PSD data were collected in four separate regions and stitched together later.

ing that the ion is stable. In our earlier report we had suggested that the cage of $\mathrm{Mo}_{13} \mathrm{~S}_{25}$ has a cavity of the size $4.5 \AA$. That cavity can accommodate the Mo atom to form $\mathrm{Mo}_{14} \mathrm{~S}_{25}{ }^{-}$. A distinct aspect of the spectrum is the presence of features assigned to $\mathrm{Mo}_{20} \mathrm{~S}_{41}$, which could be a fullerene-like cage with an $\mathrm{S}$ atom inside. The ion intensity was inadequate to examine this structural suggestion in detail by PSD analysis.

In $\mathrm{Mo}_{13} \mathrm{~S}_{25}$, the core of Mo atoms resembles a curled triangular net of Mo atoms, which remains largely unmodified during the structural relaxation, whereas $\mathrm{Mo}_{13} \mathrm{~S}_{28}$ has the shape of a short cylinder, revealing significant structural relaxation and deviation from the initial spherical cage-like structure. This indicates that these clusters may have formed by curling of the $\mathrm{MoS}_{2}$ sheets after the removal S atoms from one side.

Wannier function analysis of the real-space electronic distribution reveals a rich bonding scenario due to a variety of bond multiplicities in both small and large clusters. Electrons relevant to chemical bonding are mainly from the $3 \mathrm{~s}$ and $3 \mathrm{p}$ orbitals of $\mathrm{S}$ and $4 \mathrm{~d}$ orbitals of Mo. In particular, the $4 \mathrm{~d}$ electrons cannot be easily detected through a simple charge density or wave function analysis, instead calling for Wannier functions description. We first discuss the localized Wannier orbitals in the small Mo-S clusters. Wannier functions describing the Mo-S bonding orbitals (Figure $7 \mathrm{a}-\mathrm{c}$ ) indicate single, double, and triple $\mathrm{Mo}-\mathrm{S}$ bonds in $\mathrm{Mo}_{2} \mathrm{~S}_{3}, \mathrm{MoS}_{3}$, and MoS clusters, respectively. This is reflected in corresponding Mo-S bond lengths of $2.38,2.12$, and $2.10 \AA$ in these clusters, respectively. Because the corresponding Wannier centers along the 

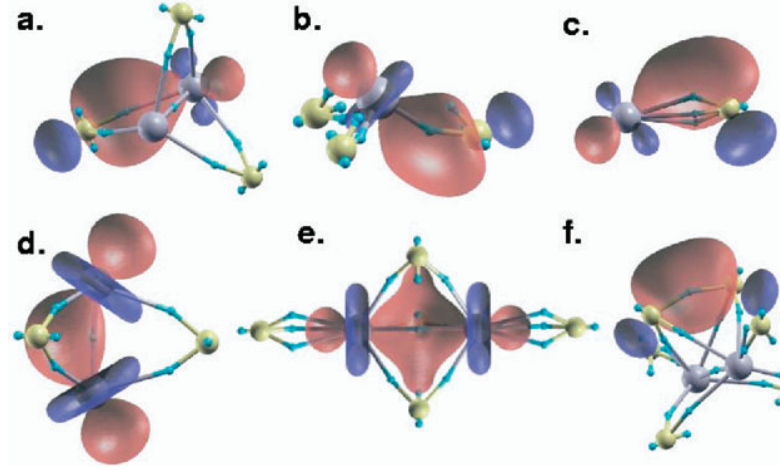

f.

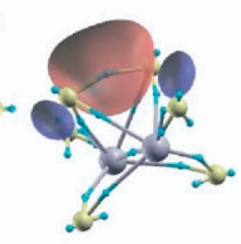

Figure 7. Localized bonding orbitals and their centers (showed as cyan-colored balls) exemplifying the different kinds of bonding in small $\mathrm{Mo}_{\mathrm{m}} \mathrm{S}_{\mathrm{n}}$ clusters: $\mathrm{Mo}-\mathrm{S}$ single, double, and triple bonds in (a), (b), and (c), respectively, Mo-Mo triple and single bonds in (d) and (e) respectively, and S-S $\sigma$ bond in (f).

Mo- $\mathrm{S}$ bonds are nearer to $\mathrm{S}$ atoms, they are characterized as polar covalent bonds. These orbitals exhibit a trend: the angle between the axis of atomic orbital and the line joining Mo and $\mathrm{S}$ atoms increases with the multiplicity of the bond, as reflected in the fact that the respective bond centers are off the $\mathrm{Mo}-\mathrm{S}$ axis.

Mo-Mo bonds (Figure 7d and e) present in the $\mathrm{Mo}_{2} \mathrm{~S}_{3}, \mathrm{Mo}_{2} \mathrm{~S}_{5}$, and $\mathrm{Mo}_{2} \mathrm{~S}_{6}$ clusters are also at the center of $\mathrm{MoS}_{n}$ polyhedra and the nature of these bonds can be understood in detail only by examining their bonding orbitals. A count of Wannier centers reveals multiplicity of Mo-Mo bonds to be one in $\mathrm{Mo}_{2} \mathrm{~S}_{5}$ and $\mathrm{Mo}_{2} \mathrm{~S}_{6}$ and three in $\mathrm{Mo}_{2} \mathrm{~S}_{3}$. Wannier functions representing the $\mathrm{Mo}$ - Mo single bond in $\mathrm{Mo}_{2} \mathrm{~S}_{6}$ and $\mathrm{Mo}_{2} \mathrm{~S}_{5}$ actually have a multicentered character, reflected in the iso-surfaces pointing toward the sulfur atoms in the equatorial plane. In contrast, orbitals of the triple bond between Mo atoms of $\mathrm{Mo}_{2} \mathrm{~S}_{3}$ constitute primarily two-centered bonds. It is expedient at this point to note that $\mathrm{Mo}_{2} \mathrm{~S}_{3}$ cluster has a more enhanced stability than that of the rest of the small clusters due to two main reasons: (1) all its $\mathrm{Mo}-\mathrm{S}$ bonds are single bonds and (2) it has triple Mo-Mo bonds, each of which is strongly covalent.

As the proportion of $S$ atoms in these clusters increases, $\mathrm{S}-\mathrm{S}$ bond (Figure $7 \mathrm{f}$ ) appears, as exhibited in the $\mathrm{Mo}_{2} \mathrm{~S}_{6}$ cluster. The Wannier function representing the $\mathrm{S}-\mathrm{S}$ bond shows a $\sigma$-bonding character made of $\left(s+p_{x}\right)$ hybridized orbitals. The lone-pair orbitals are centered in the close vicinity of the $\mathrm{S}$ atoms. Both these two types of orbitals typically have an axis pointing away from the rest of the atoms in the cluster.

Similar to those in small clusters, Wannier centers (Figure 8a) of larger clusters indicate predominantly covalent bonding between the atoms. We present here a comparative bonding analysis of $\mathrm{Mo}_{13} \mathrm{~S}_{25}$ and $\mathrm{Mo}_{14} \mathrm{~S}_{25}$ clusters. Whereas the lone pairs (Figure $8 \mathrm{~b}$ ) on each of the $S$ atoms in the outer shell point radially outward, their sp hybridized orbitals mix with the $4 \mathrm{dz}^{2}$-type orbitals of Mo in the inner shell to form heteropolar covalent S-Mo bonds. These sp hybridized orbitals also form homopolar S - S $v$ bonds in the three nearestneighbor pairs of sulfur atoms in the outer shell. Interestingly, we find multicentered covalent bonds (Figure $8 \mathrm{c}$ and d) formed inside the cage enclosed by the inner shell of the Mo atoms. These bonds, formed mainly by the delocalized $5 \mathrm{~s}$ and the $4 \mathrm{~d}$ electrons of Mo, can be useful in explaining the extraordinary stability of the caged structure. These multicentered bonds are essential for the MoS layer, formed by the removal of all the $\mathrm{S}$ atoms from one side of the $\mathrm{MoS}_{2}$ layer in the bulk, to curl up and eventually form a closed-cage structure. In $\mathrm{Mo}_{14} \mathrm{~S}_{25}$, an extra Mo atom inside the cage is attached to the Mo atoms in the inner shell mainly through the multicentered covalent bonds (Figure 8d) formed by the $4 \mathrm{~d}$ electrons. Thus, the two centered interlayer single bonds (between the Mo layer and the S layer) seem to provide stability to the shell structure, whereas the multicentered bonds seem to be crucial in making the shell curl and enclose a volume. This explains the greater stability of $\mathrm{Mo}_{14} \mathrm{~S}_{25}$ than that of $\mathrm{Mo}_{13} \mathrm{~S}_{25}$.

\section{Summary and Conclusions}

In summary, we have explored the novel closed-cage clusters formed in the gas phase, derived from nanoflakes of $\mathrm{MoS}_{2}$ synthesized from elemental precursors. These clusters are likely to be inorganic fullerenes and may be formed by the curling of nanoflakes of $\mathrm{MoS}_{2}$. We find that such clusters are not formed from stable molybdenum oxide clusters. The presence of molybdenum sulfide clusters in the MALDI-MS analy-
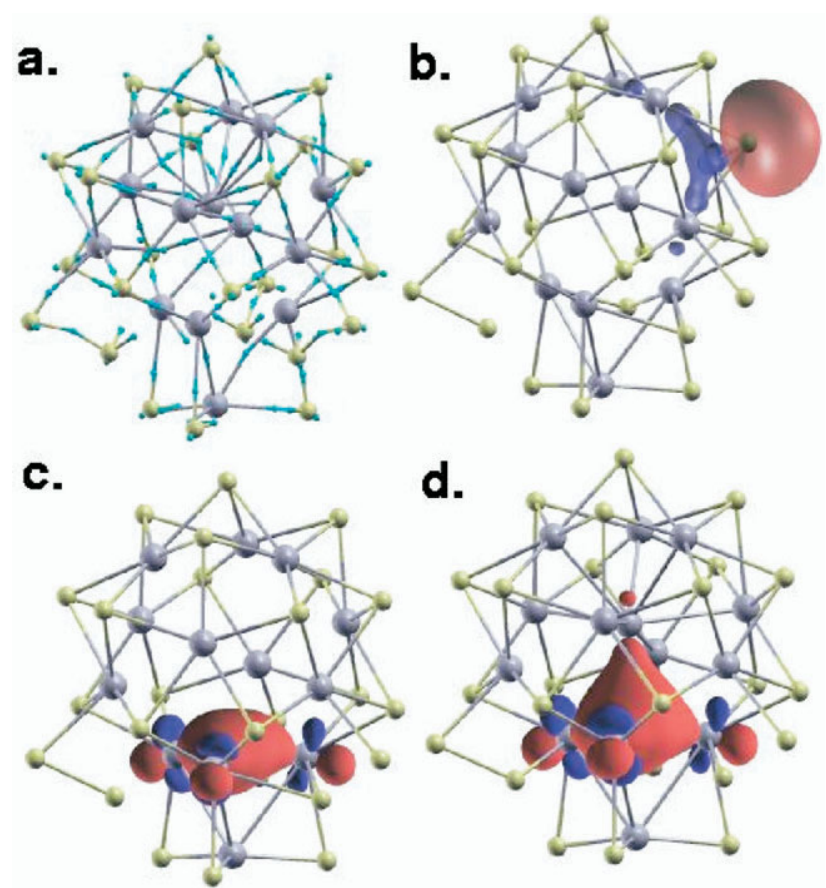

Figure 8. Localized bonding orbitals and their centers (shown as cyan-colored balls) in large clusters: orbital centers in $\mathrm{Mo}_{14} \mathrm{~S}_{25}$ in (a), a lone pair orbital in (b), similar multicentered bonds in $\mathrm{Mo}_{13} \mathrm{~S}_{25}$, and $\mathrm{Mo}_{14} \mathrm{~S}_{25}$ clusters in (c) and (d), respectively. 
sis suggests their existence in the condensed phase. Our computational work reveals the possible structure of these closed cages and confirms their stability. We find a rich variety in terms of centricity and multiplicity of $\mathrm{Mo}-\mathrm{Mo}, \mathrm{S}-\mathrm{Mo}$, and $\mathrm{S}-\mathrm{S}$ bonds in $\mathrm{Mo}_{\mathrm{m}} \mathrm{S}_{\mathrm{n}}$ clusters.

\section{Acknowledgments}

Our nanomaterials program is supported by the Department of Science and Technology. D. M. D. J. Singh was supported by a Swarnajayanti Fellowship awarded to T. Pradeep.

\section{References}

1. Guo, B. C.; Kerns, K. P.; Castleman, A. W., Jr. $\mathrm{Ti}_{8} \mathrm{C}_{12}{ }^{+}$MetalloCarbohedrenes: A New Class of Molecular Clusters? Science 1992, 255 , 1411-1413.

2. Rohmer, M.-M.; Bénard, M.; Poblet, J.-M. Structure, Reactivity, and Growth Pathways of Metallocarbohedrenes $\mathrm{M}_{8} \mathrm{C}_{12}$ and Transition Metal/Carbon Clusters and Nanocrystals: A Challenge to Computational Chemistry. Chem. Rev. 2000, 100, 495-542.

3. Tenne, R.; Margulis, L.; Genut, M.; Hodes, G. Polyhedral and Cylindrical Structures of Tungsten Disulphide. Nature 1992, 360, 444-446.

4. Rapoport, L.; Bilik, Y.; Feldman, Y.; Homyonfer, M.; Cohen, S. R.; Tenne, R. Hollow Nanoparticles of $\mathrm{WS}_{2}$ as Potential Solid-state Lubricants. Nature 1997, 387, 791-793.

5. Zak, A.; Feldman, Y.; Alperovich, V.; Rosentsveig, R.; Tenne, R. Growth Mechanism of $\mathrm{MoS}_{2}$ Fullerene-Like Nanoparticles by the Gas Phase Synthesis. J. Am. Chem. Soc. 2006, 122, 11108-11116.

6. Schuffenhauer, C.; Parkinson, B. A.; Jin-Phillipp, N. Y.; Joly-Pottuz, L.; Martin, J. M.; Popovitz-Biro, R.; Tenne, R. Synthesis of Fullerene-Like Tantalum Disulfide Nanoparticles by Gas-phase Reaction and Laser Ablation. Small 2005, 1, 1100-1109.

7. Sen, R.; Govindaraj, A.; Suenaga, K.; Suzuki, S.; Kataura, H.; Iijima, S.; Achiba, Y. Encapsulated and Hollow Closed-cage Structures of $\mathrm{WS}_{2}$ and $\mathrm{MoS}_{2}$ Prepared by Laser Ablation at $450-1050^{\circ} \mathrm{C}$. Chem. Phys. Lett. 2001, 340, 242-248.

8. Chick, K. Y.; Nath, M.; Parkinson, B. A. TaS ${ }_{2}$ Nanoplatelets Produced by Laser Ablation. J. Mater. Res. 2006, 21, 1243-1247.

9. José-Yacamán, M.; López, H.; Santiago, P.; Galván, D. H.; Garzón, I. L.; Reyes, A. Studies of $\mathrm{MoS}_{2}$ Structures Produced by Electron Irradiation. Appl. Phys. Lett. 1996, 69, 1065-1067.

10. Duphil, D.; Bastide, S.; Lévy-Clément, C. Chemical Synthesis of Molybdenum Disulfide Nanoparticles in an Organic Solution. J. Mater. Chem. 2002, 12, 2430-2432

11. Bastide, S.; Lévy-Clément, C.; Albu-Yaron, A.; Boucher, A.-C.; AlonsoVante, N. MoSe, Nanocrystallites Synthesized at Low Temperature via a Chemical Solution Route. Electrochem. Solid State Lett. 2000, 3, 450-451.

12. Ponomarev, E. A.; AlbuYaron, A.; Tenne, R.; Lévy-Clément, C. Electrochemical Deposition of Quantized Particle $\mathrm{MoS}_{2}$ Thin Films. J. Electrochem. Soc. 1997, 144, 277-279.

13. Suslick, K. S.; Hyeon, T.; Fang, M. Nanostructured Materials Generated by High-intensity Ultrasound: Sonochemical Synthesis and Catalytic Studies. Chem. Mater. 1996, 8, 2172-2179.

14. Uzcanga, I.; Bezverkhyy, I.; Afanasiev, P.; Scott, C.; Vrinat, M. Sonochemical Preparation of $\mathrm{MoS}_{2}$ in Aqueous Solution: Replication of the Cavitation Bubbles in an Inorganic Material Morphology. Chem. Mater. 2005, 17, 3575-3577.

15. Mdleleni, M. M.; Hyeon, T.; Suslick. K. S. Sonochemical Synthesis of Nanostructured Molybdenum Sulfide. J. Am. Chem. Soc. 1998, 120, $6189-6190$.
16. Dhas, N. A.; Gedanken, A. Sonochemical Synthesis of Molybdenum Oxide- and Molybdenum Carbide-Silica Nanocomposites. Chem. Mater. 1997, 9, 3144-3154.

17. Sostaric, J. Z.; Caruso-Hobson, R. A.; Mulvaney, P.; Grieser, F. Ultrasound-induced Formation and Dissolution of Colloidal CdS. J. Chem. Soc. Faraday Trans. 1997, 93, 1791-1795.

18. Avivi, S.; Mastai, Y.; Gedanken, A. A New Fullerene-like Inorganic Compound Fabricated by the Sonolysis of an Aqueous Solution of $\mathrm{TlCl}_{3}$. J. Am. Chem. Soc. 2000, 122, 4331-4334.

19. James, M. L.; Mann, H. A.; Wu, M.; Johnson, P. M.; White, M. G. Gas-phase Production of Molybdenum Carbide, Nitride, and Sulfide Clusters and Nanocrystallites. J. Phys. Chem. B 2003, 107, 10359-10366.

20. Bertram, N.; Cordes, J.; Kim, Y. D.; Ganteför, G.; Gemming, S.; Seifert, G. Nanoplatelets Made from $\mathrm{MoS}_{2}$ and $\mathrm{WS}_{2}$. Chem. Phys. Lett. 2006, 418, 36-39.

21. Gemming, S.; Tamuliene, J.; Seifert, G.; Bertram, N.; Kim, Y. D Gantefor, G. Electronic and Geometric Structures of $\mathrm{Mo}_{\mathrm{x}} \mathrm{S}_{\mathrm{y}}$ and $\mathrm{W}_{\mathrm{x}} \mathrm{S}_{\mathrm{y}}(\mathrm{x}$ $=1,2,4 ; \mathrm{Y}=1-12$ ) clusters. Appl. Phys. A 2006, 82, 161-166.

22. Seifert, G.; Tamuliene, J.; Gemming, S. $\mathrm{Mo}_{\mathrm{n}} \mathrm{S}_{2 \mathrm{n}+\mathrm{x}}$ Clusters, Magic Numbers, and Platelets. Comput. Mater. Sci. 2006, 35, 316-320.

23. Bertram, N. Kim, Y. D.; Ganteför, G. Sun, Q.; Jena, P.; Tamuliene, J. Seifert, G. Experimental and Theoretical Studies on Inorganic Magic Clusters: $\mathrm{M}_{4} \mathrm{X}_{6}(\mathrm{M}=\mathrm{W}, \mathrm{Mo} ; \mathrm{X}=\mathrm{O}, \mathrm{S})$. Chem. Phys. Lett. 2004, 396, 341-345.

24. Lauritsen, J. V.; Kibsgaard, J.; Helveg, S.; Topsøe, H.; Clausen, B. S.; Lægsgaard, E.; Besenbacher, F. Size-dependent Structure of MoS Nanocrystals. Nat. Nanotechnol. 2007, 2, 53-58 and references cited therein

25. Parilla, P. A.; Dillon, A. C.; Parkinson, B. A.; Jones, K. M.; Alleman, J.; Riker, G.; Ginley, D. S.; Heben, M. J. Formation of Nanooctahedra in Molybdenum Disulfide and Molybdenum Diselenide Using Pulsed Laser Vaporization. J. Phys. Chem. B 2004, 108, 6197-6207.

26. Singh, D. M. D. J.; Pradeep, T.; Bhattacharjee, J.; Waghmare, U. V. Novel Cage Clusters of $\mathrm{MoS}_{2}$ in the Gas Phase. J. Phys. Chem. A 2005, 109, 7339-7342.

27. Singh, D. M. D. J.; Pradeep, T. $\mathrm{Te}_{n} \mathrm{~S}_{4}{ }^{+}(\mathrm{n}=1-4)$ Clusters in the Gas Phase. Chem. Phys. Lett. 2005, 417, 34-38.

28. Singh, D. M. D. J.; Pradeep, T. $\mathrm{Mo}(\mathrm{W})_{\mathrm{n}} \mathrm{O}_{\mathrm{m}}{ }^{ \pm}(\mathrm{n}=1-33 ; \mathrm{m}=2-75)$ Clusters in the Gas Phase. Chem. Phys. Lett. 2004, 395, 351-355.

29. Coehoorn, R.; Haas, C.; de Groot, R. A. Electronic Structure of $\mathrm{MoSe}_{2}$ $\mathrm{MoS}_{2}$, and WSe 2 . II. The Nature of the Optical Band Gaps. Phys. Rev. B 1987, 35, 6203-6206.

30. Wilcoxon, J. P.; Samara, G. A. Strong Quantum-Size Effects in a Layered Semiconductor: MoS 2 Nanoclusters. Phys. Rev. B 1995, 51, 7299-7302.

31. de Laeter, J. R.; Böhlke, J. K.; De Bièvre, P.; Hidaka, H.; Peiser, H. S.; Rosman, K. J. R.; Taylor, P. D. P. Atomic Weights of the Elements. Review 2000. Pure Appl. Chem. 2003, 75, 683-800.

32. Baroni, S.; Corso, A. D.; Gironcoli, S.; Giannozzi, P. http://www. pwscf.org.

33. Perdew, J. P.; Burke, K.; Ernzerhof, M. Generalized Gradient Approximation Made Simple. Phys. Rev. Lett. 1996, 77, 3865-3868.

34. Vanderbilt, D. Soft Self-consistent Pseudopotentials in a Generalized Eigenvalue Formalism. Phys. Rev. B 1990, 41, 7892-7895.

35. Bhattacharjee, J.: Waghmare, U. V. Localized Orbital Description of Electronic Structures of Extended Periodic Metals, Insulators, and Confined Systems: Density Functional Theory Calculations. Phys. Rev. B 2006, 73, 121102 .

36. Gonze, X. Beuken, J-M.; Caracas, R. Detraux, F. Fuchs, M. Rignanese, G.-M.; Sindic, L.; Verstraete, M.; Zerah, G.; Jollet, F.; Torrent, M.; Roy, A.; Mikami, M.; Ghosez, Ph.; Raty, J.-Y.; Allan, D. C. First-Principles Computation of Material Properties: The ABINIT Software Project. Comput. Mater. Sci. 2002, 25, 478-492.

37. Zheng, X.; Zhu, L.; Yan, A.; Bai, C.; Xie, Y. Ultrasound-assisted Cracking Process to Prepare $\mathrm{MoS}_{2}$ Nanorods. Ultrason. Sonochem. 2004, 11, 83-88.

38. Yamase, T.; Ishikawa, E.; Abe, Y.; Yano, Y. Photoinduced Self-assembly to Lanthanide-Containing Molybdenum-Blue Superclusters and Molecular Design. J. Alloys Compd. 2006, 408-412, 693-700. 